\title{
Coupling and Strain Effects in Vertically Stacked Double InAs/GaAs Quantum Dots: Tight-Binding Approach
}

\author{
W. JASKÓLSKI*, M. ZIELIŃSKI \\ Instytut Fizyki UMK, Grudziądzka 5, 87-100 Toruń, Poland \\ AND G.W. BRYANT \\ National Institute of Standards and Technology \\ Gaithersburg, MD 20899-8423, USA
}

\begin{abstract}
The empirical tight-binding approach is used to study atomic-scale effects on electronic coupling in vertically stacked, self-assembled InAs/GaAs quantum dots. A model with unstrained dots is first studied to isolate the atomistic coupling effects from the strain effects. The strain effects are next considered by means of the valence force field method. Electron levels in coupled quantum dots follow closely the simple analogy of coupled dots as artificial molecules. The electron ground state of double dot has always bonding-like character. The coupling of hole states is more complicated because the coupling depends both of the hole envelope function and the atomic character of the hole state. It is shown that the character of the hole ground state of double dot changes from antibonding to bonding-like, when the distance between the dots decreases. It reorders hole levels, changes state symmetries, and makes changes in optical spectra. The calculated red-shift of the lowest transition for closely-spaced dots agrees well with experimental data. We present also some preliminary results on strain effects in such nanocrystals.
\end{abstract}

PACS numbers: 71.35.Cc

\section{Introduction}

The electronic and optical properties of self-organized quantum dots have been intensively studied for the last ten years. The ease of their fabrication, well

*corresponding author; e-mail: wj@phys.uni.torun.pl 
defined sizes and shapes, and strong quantum confinement make them attractive for applications as optoelectronic devices. Of special interest are arrays of vertically stacked self-assembled quantum dots (QDs) [1-4] since one expects enhancement in the performance of quantum dot lasers if many layers of nanodots are employed in such devices.

When the layers of dots are closely spaced, the electronic coupling between vertically stacked quantum dots can lead to a mixing of states and level splittings that change the structure of optically active transitions. It offers the possibility of tailoring optical properties of quantum dot light-emitting devices by changing the interdot distance. If the dots are closely and regularly spaced, then coherent electronic coupling across the entire array of dots should be possible. This opens up the possibility of using dots as the artificial atoms or building blocks for entirely new artificial solids [5]. Quantum dots and especially coupled dots are thought also as good candidates for realizing nanoscale qubits. In each of the cases mentioned, it is important to understand the nature of the electronic coupling between the quantum dots. In this paper we investigate this coupling.

The first step toward understanding the physics of arrays of vertically stacked self-assembled dots is to study systems of two coupled dots. Systems of coupled dots have been recently investigated theoretically by many authors [6-15], where effective mass approaches were mainly used. For precise engineering of the level structure in coupled-dot systems, accurate inclusion of atomic-scale effects is also necessary. This is especially important for small quantum dots and when the thickness of the separating medium spacer layer is only a few monolayers (ML). The tight-binding (TB) approach should give a description of the coupling between the dots that includes the atomic-scale effects ignored in effective mass theories. The main aim of our work is to study how atomic-scale effects influence the electronic and optical properties of stacked QDs by using an atomistic TB approach.

In this paper we investigate systems of two self-assembled, vertically stacked, quantum dots. We perform tight-binding calculations first for unstrained coupled dots and next for strained single dot. It allows us to investigate the role of coupling and strain separately.

\section{Theory}

To calculate one-electron states in systems of coupled quantum dots we use the tight-binding method [16-19]. Recently, we used the TB approach to successfully explain (i) optical spectra and the appearance of dark excitons in multishell quantum dot-quantum wells [20,21] and (ii) coupling effects in artificial molecules and close-packed arrays formed by chemically synthesized nanocrystals [22-24]. It has been shown [20] that level ordering, splitting, and degeneracy can depend on atomic-scale details, like the definition of quantum dot center (at an anion, a cation, or at midbond). Continuous effective medium models completely miss these effects. 
In our TB approach we assume that atoms occupy the sites of a regular zinc-blende lattice. Each atom is described by its outer valence $s$ orbital, the 3 outer $p$ orbitals, and an additional $s^{*}$ orbital that accounts for higher lying states [20]. Only coupling of on-site and nearest neighbor orbitals is included. Empirical parameters are obtained by adjusting the matrix elements of the TB Hamiltonian to reproduce experimentally known band gaps and effective masses of the bulk band structures [25]. The valence band offset between the InAs and GaAs is taken as $0.2 \mathrm{eV}$.

We investigate two vertically stacked InAs lens-shaped quantum dots situated on separate parallel wetting layers, and embedded in a large cylindrical GaAs box (see Fig. 1). The cylindrical shape of the GaAs box ensures that the symmetry of quantum dot states is not affected by the geometry of the wetting layer (WL) and GaAs box. The size of the external box is chosen to ensure that further increases in box size change the bound-state energy spectra by only a few meV without changing the level structure qualitatively. Surface states of the GaAs box are excluded by passivating the surface dangling bonds (by shifting their energies high above the conduction band so that they do not modify states near the band gap). GaAs spacer layers, separating the top of the lower quantum dot from the bottom of the upper WL, with thickness from 0 to $20 \mathrm{ML}$ are considered.

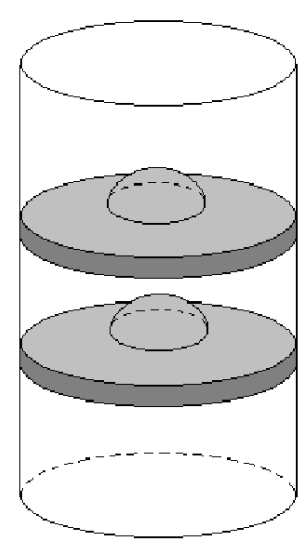

Fig. 1. Schematic for the system of two InAs vertically stacked InAs lens-type QDs on wetting layers embedded in a GaAs cylinder. The lengths are not drawn to scale.

The states are found by diagonalizing the TB Hamiltonian using an iterative eigenvalue solver [26]. Only the electron and hole states with energies close to the InAs conduction band and valence band edges are found.

The single-particle electron-hole transition rates are calculated by evaluating the dipole matrix elements in real space using the TB wave functions (the details are given in Ref. [20]). The symmetries of the electron and hole states determine which electron-hole transitions are optically active. 


\section{Results and discussion}

The investigated system is shown schematically in Fig. 1. The wetting layers are 2 ML thick and are parallel to the (001) plane. The height of the lens is $2 a(\approx 1.2 \mathrm{~nm})$ and the base diameter is $10 a$. The diameter of the GaAs cylinder is $18 a$. There are 580 atoms in each lens-type quantum dot and 3118 atoms in each wetting layer. The GaAs/InAs system has more than 41000 atoms in the largest case investigated. Although the investigated lenses are a bit smaller than the self-assembled InAs/GaAs QDs that are typically grown, the essential physics of the coupled nanocrystals should not depend critically on the dot size. Moreover, smaller dots should be considered for cases where coupling effects are to be exploited. Coupling effects should be larger for smaller dots because the confined states are less tightly bound in smaller dots.

\subsection{Energy levels}

The effective energy gap between the lowest hole level and the lowest electron level for the smallest pure GaAs cylinder considered, of radius $10 a$ and height $10 a$, is $1.68 \mathrm{eV}$. The effective energy gap of a system containing only the InAs wetting layer embedded in a GaAs box is $1.40 \mathrm{eV}$. The absolute zero of the energy scale is at the top of the bulk InAs valence band (VB). The semi-2D InAs WL electron continuum starts at about $1.20 \mathrm{eV}$ and contains 15 discrete states. The corresponding hole continuum starts at about $-0.19 \mathrm{eV}$ and contains more than 20 energy levels before reaching the GaAs VB edge threshold. All these states belong to a single WL subband since their wave functions do not have nodal planes parallel to the WL.

To understand coupling effects, we first must understand the bound states of a single QD on a WL. For the sizes that we consider here, the single quantum dot on a WL has three bound electron energy levels: a nondegenerate ground state and two higher, almost degenerate levels (the energy difference $\approx 1 \mathrm{meV}$ ). Seven hole states are bound. When no QD is present and the GaAs cylinder is vertically symmetric, some WL states, including the hole ground state, are doubly degenerate. This degeneracy is lifted in the WL+QD system. This suggests that the splitting of the ground hole state is connected inherently with the vertical asymmetry of the WL+QD system.

For the single lens considered here, the lowest electron state is symmetric in the $X Y$-plane. The excited bound states have global $p$ symmetry (resulting from hemispherical geometry of the QD). They are made primarily from $s$ cation orbitals with a $p$-like envelope function. A weak mixing of $p$ atomic orbitals into these $p$-type electron states lifts slightly $(1 \mathrm{meV})$ the degeneracy of the states. As a result, the lowest excited state is polarized along (110).

The two lowest hole states have densities distributed along (110) for the ground hole state and (110) for the first excited hole state and are nondegenerate with a splitting of about $3 \mathrm{meV}$. This polarization of the hole ground state does 
not depend on whether the QD base is centered at an anion or cation. When one considers the identical lens pointing down rather than up, the ground state density is rotated by 90 degrees. It means that the polarization of the hole ground state follows the bond direction defined between atoms on the lowest plane of atoms inside the dot and the atoms on the next plane inside the dot. This polarization is an atomic-scale feature that would not be predicted by effective mass theories. The polarization splitting should not be ignored, because, as we will show later, the splittings due to polarization and coupling are comparable.

Inside an isolated InAs dot, the two lowest hole states are made primarily from $p_{x}$ and $p_{y}$ As atomic orbitals with a small contribution from As $p_{z}$ atomic orbitals. These hole states are analogous to quantum-well heavy-hole states. Parity in the $z$-direction is not an exact symmetry because the lens does not have exact inversion symmetry about any $X Y$-plane. Nonetheless, total $z$-parity is an approximate symmetry that is useful for characterizing these states. Here, the total $z$-parity of a state is the $z$-parity of the full wave function of the state, as determined for each type of atomic orbital that contribute to the state, by the $z$-parity of that atomic orbital, and the z-parity of the envelope function for that type of orbital. The two lowest hole states have even total $z$-parity relative to an $X Y$-plane in the WL-dot region. For these two hole states, the As $p_{x}$ and $p_{y}$ orbitals have envelope functions with even $z$-parity and the smaller As $p_{z}$ component has an envelope function with odd $z$-parity.

For two vertically stacked dots, we define the separation between dots $d$ as the distance between the top of the lower dot and the bottom of the upper WL. For well separated dots, i.e., when $d=10 a(\approx 20 \mathrm{ML})$, the electron bound levels

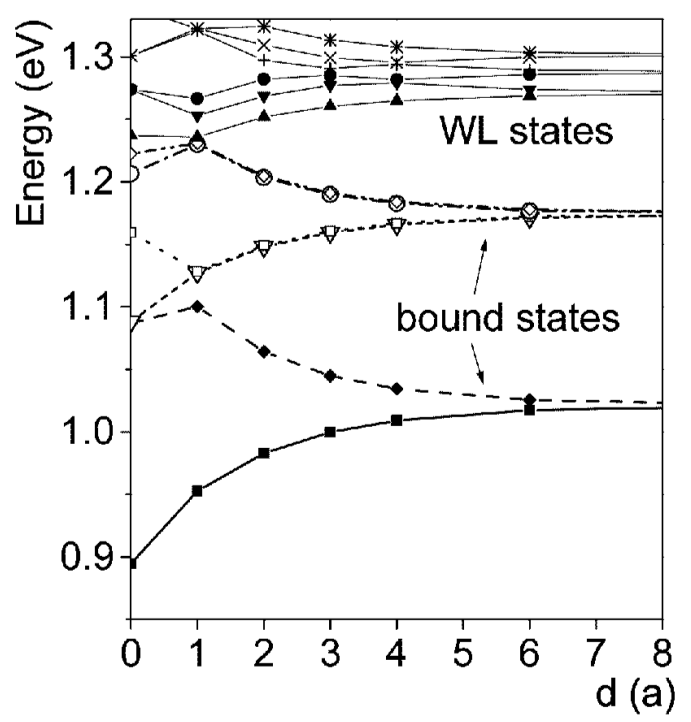

Fig. 2. Several lowest electron energy levels of two lens-type QDs vs. distance $d$. The $\mathrm{WL}$ continuum starts at $\approx 1.2 \mathrm{eV}$. 
are almost doubly degenerate (within $2 \mathrm{meV}$ ). When the separation decreases, the energy levels split and can cross. The electron energy levels versus separation $d$ are shown in Fig. 2. Several lower WL states are also shown. The lowest hole energy levels are shown in Fig. 3. The plots of the corresponding density isosurfaces (60\% of the total density) are shown in Figs. 4 and 5 , respectively ${ }^{\dagger}$.

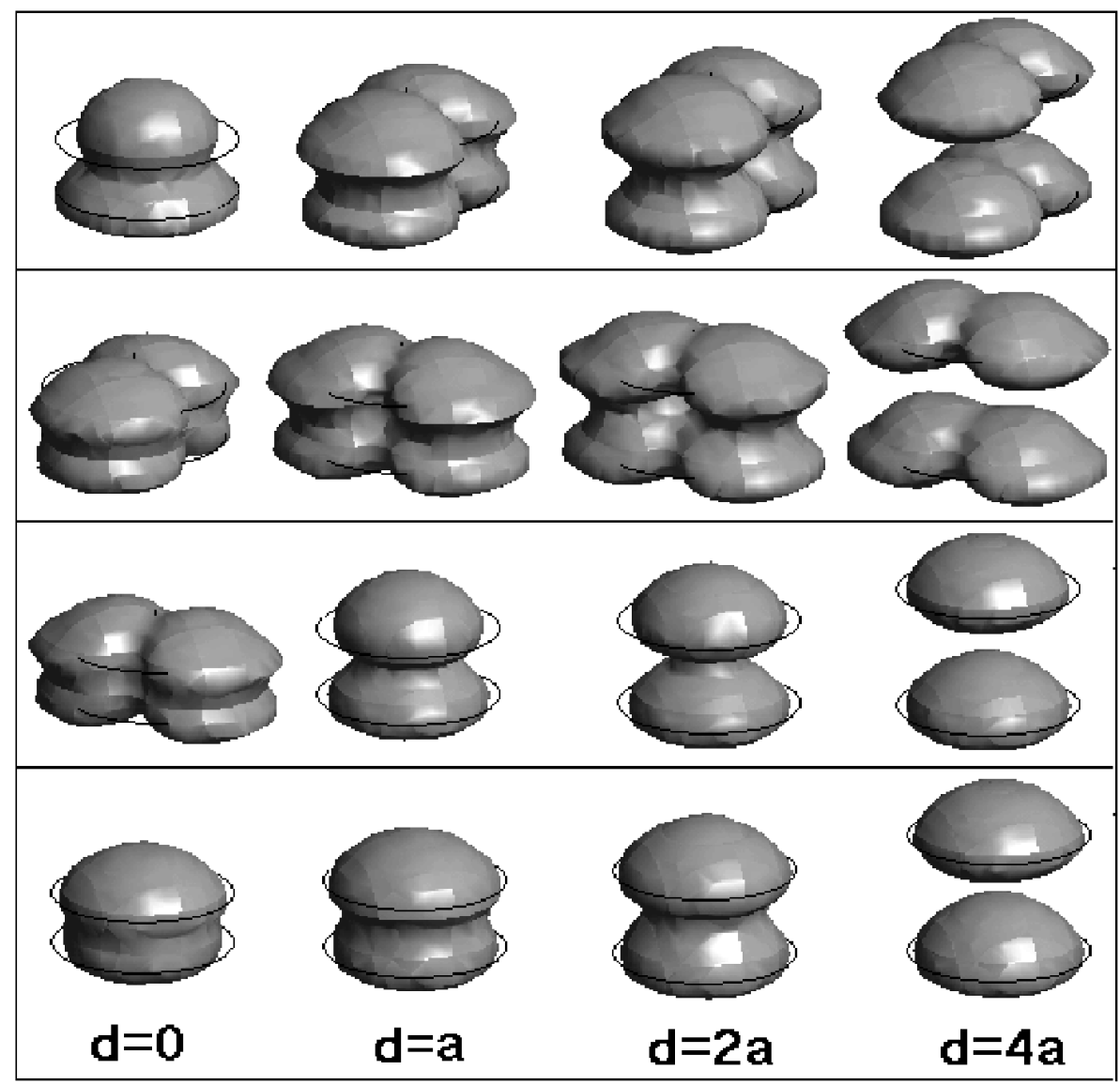

Fig. 3. Density isosurfaces $(60 \%)$ of the four lowest electron energy levels (from bottom to top) of two vertically stacked InAs lens-type QDs vs. distance $d$.

When $d$ decreases, the quasi-degeneracy of the $p$-type electron states is not lifted, even for $d=0$. For $d<1 a$ the energy splitting of the symmetric and antisymmetric combinations is so strong that the upper $s$-type level is pushed above the lower pair of $p$-type energy levels. For $d=0$, the three lowest energy

$\dagger$ Since the investigated lenses are smaller than the QDs studied in [27], the dot states we calculate are less strongly bound and the calculated density isosurfaces $(60 \%)$ more diffused than those in [27]. 


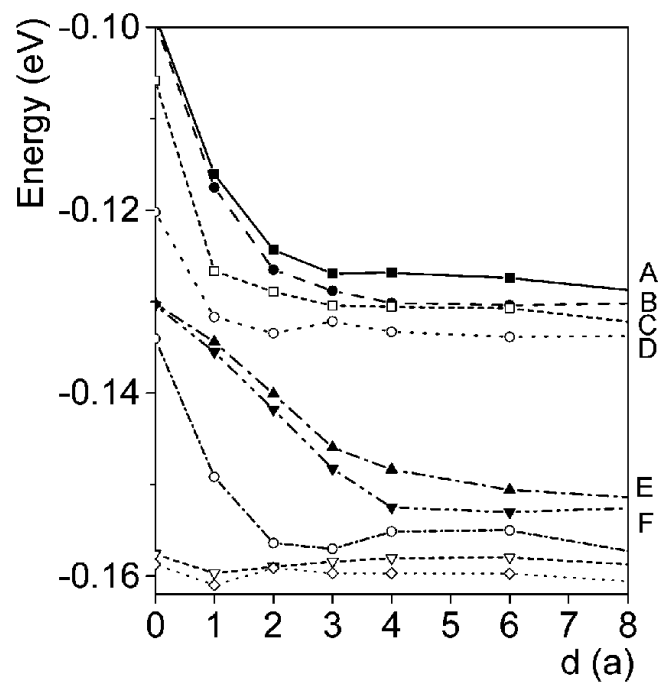

Fig. 4. Several lowest hole energy levels of two lens-type QDs vs. distance $d$. The WL continuum starts at $\approx-0.19 \mathrm{eV}$.

levels are: (1) nondegenerate bonding-like $s$-type level, (2) twofold degenerate bonding-like $p$-type energy level and (3) nondegenerate antibonding-like $s$-type level. The states (1) and (2) would convert into the bound states of a single QD if the dots were allowed to overlap.

The hole states undergo a complex reorderings of levels and symmetry changes for decreasing separation $d$. The spectrum of hole energy levels versus $d$ is shown in Fig. 3. The individual states of the single lens become doublets of states, formed approximately by the symmetric and antisymmetric combinations of the individual dot states when two equivalent dots are coupled ${ }^{\ddagger}$. For well separated dots, as for a single quantum dot, the two lowest doublets of states have densities distributed along (11̄0) and (110) (see Fig. 5) and are not degenerate. As for isolated dots, the first two pairs in double-dot artificial molecule are made primarily from $p_{x}$ and $p_{y}$ As orbitals with only a small As $p_{z}$ contribution. The contribution of the As $p_{x}$ and $p_{y}$ orbitals decay rapidly away from the dots because they come from the heavy-hole component. Far from the dots, the remaining contribution is the light-hole component from the As $p_{z}$ orbitals. As a consequence, the coupling is mediated by As $p_{z}$ orbitals when the dots are widely separated. The lowest single-dot hole states have even total $z$-parity inside each dot, but the envelope for the As $p_{z}$ component has odd $z$-parity inside each dot. When the two dots are widely spaced, an antisymmetric combination of the single-dot states of the two dots is needed to provide an envelope for the As $p_{z}$ component of the

\footnotetext{
$\ddagger$ The TB wave function (as a vector of coefficients) is not a continuous function. Thus, isosurfaces of high densities, calculated within the marching cubes algorithm, may not fully reflect the existing nodal surfaces.
} 


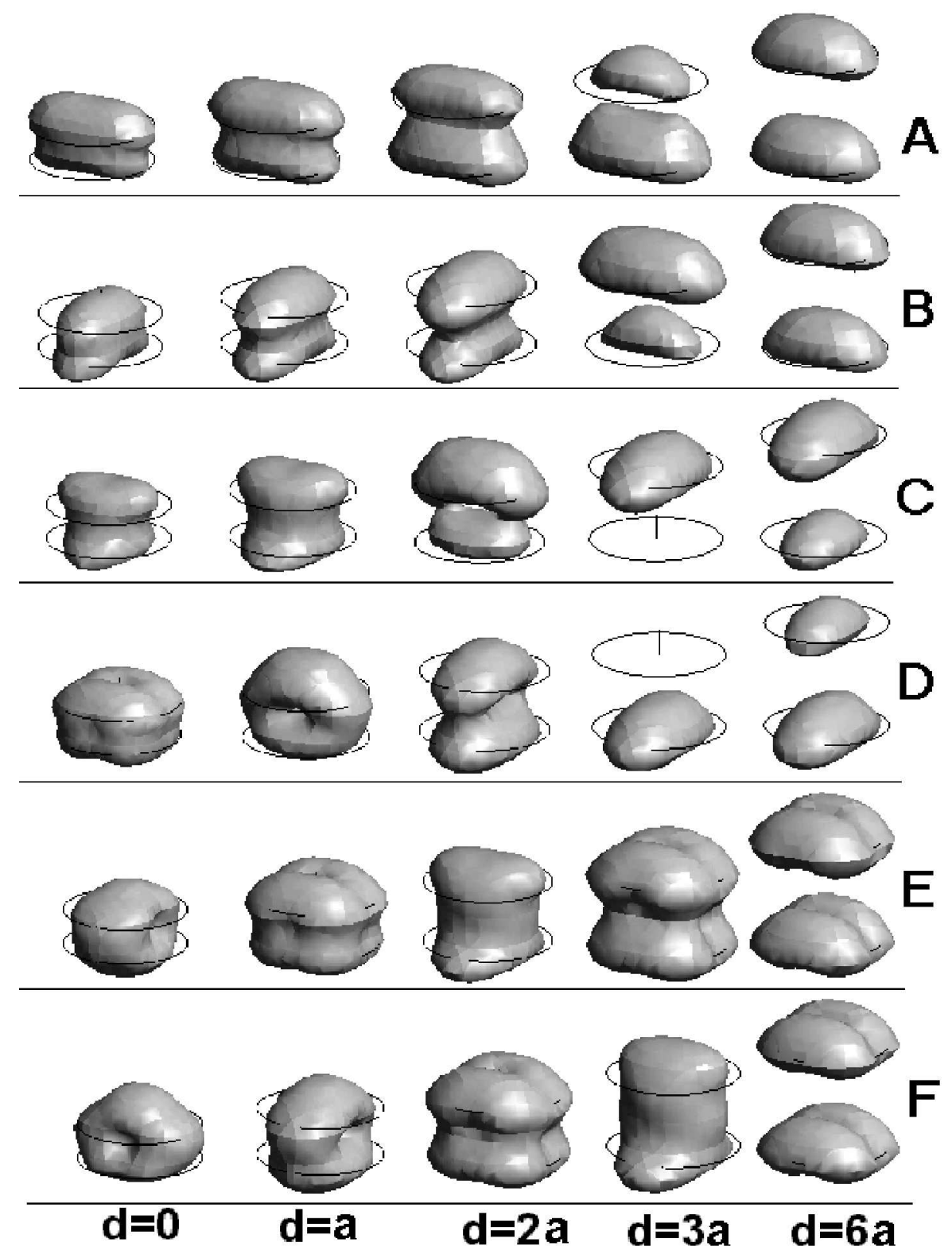

Fig. 5. Density isosurfaces (60\%) of six lowest hole energy levels (from top to bottom) of two InAs lens-type QDs vs. distance $d$.

double-dot state that remains finite between the dots. Thus, antisymmetric mixing provides the strongest coupling, and gives the lower energy state of the doublet. When the dots are close together $(d \approx 2 a)$, the coupling is mediated by the As $p_{x}$ 
and $p_{y}$ orbitals that are dominant just outside the dots. As a consequence, the character of the ground hole state changes from antisymmetric to symmetric.

Additionally, for $d$ where coupled levels cross, states can be localized strongly in one dot or the other, as happens for $d=3 a$. This localization occurs because the weak symmetry breaking due to the dot shape and placement of the WL is larger than the splitting due to coupling.

\subsection{Transition rates}

Near band-edge optical transition rates for a single lens-type dot and two vertically stacked, lens-type QDs with $d=4 a, a, 0$ are shown in Fig. 6. The atomic dipole moments used for these calculations are: $\left\langle s|x| p_{x}\right\rangle=0.0925 \mathrm{~nm}$ for In, $0.0657 \mathrm{~nm}$ for As and $0.0815 \mathrm{~nm}$ for $\mathrm{Ga} ;\left\langle s^{*}|x| p_{x}\right\rangle=0.076 \mathrm{~nm}$ for In, $0.044 \mathrm{~nm}$ for As and $0.0736 \mathrm{~nm}$ for Ga [28]. Dipole moments between overlapping bond orbitals from nearest neighbors is $0.008 \mathrm{~nm}$ for both InAs and GaAs. The transition rates shown in Fig. 6 are averaged over all spatial directions.

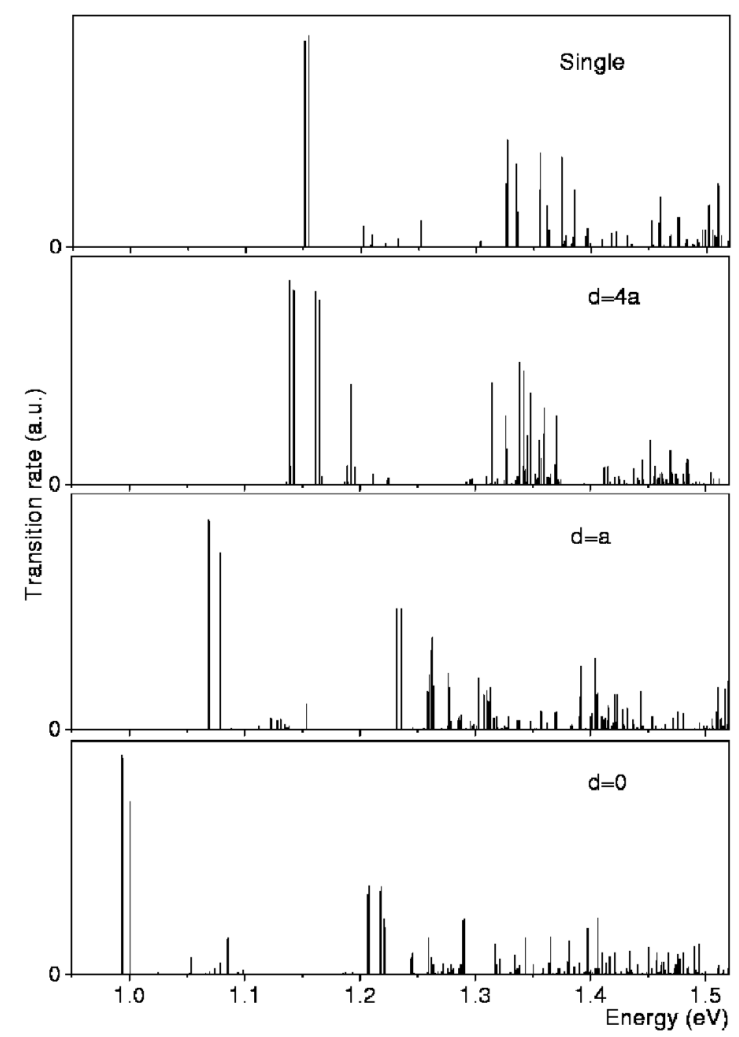

Fig. 6. Electron-hole transition rates for single and two stacked lens-type InAs QDs. 
For the single QD, only two electron states and seven hole states are bound. Thus, only limited number of bound-to-bound transitions exist below $1.23 \mathrm{eV}$, where the transitions involving WL states start. Due to symmetry, only transitions at $1.150 \mathrm{eV}, 1.155 \mathrm{eV}$, and $1.18 \mathrm{eV}$ are optically active. The lowest pair corresponds to transitions for in-plane polarization between the electron ground state and the pair of nearly degenerate hole states with densities polarized along the $(1 \overline{1} 0)$ and (110) (see Fig. 6). The next is for $z$-polarized transitions to the fourth hole state.

It is worthwhile noting that for the single QD the two lowest transitions are active only for light polarized in the WL plane. In contrast, the next active transition, corresponding to $\mathrm{e}-\mathrm{h} 4$, is active only for light polarized perpendicular to the WL plane. Due to the envelope symmetry of the third hole wave function, which has a nodal plane perpendicular to (110) (see the fifth or six hole wave function for $d=6$ ) the $\mathrm{e} 1-\mathrm{h} 3$ transition is dark in both polarizations. For some interdot separations, e.g. $d=4 a$, the lowest transition is a dark transition because the lowest hole state has antisymmetric character. The dark transition is two orders of magnitude weaker than the most active one.

A strong coupling between two slightly larger lens-type (or disk-like) self-assembled InAs/GaAs QDs was observed recently in the experiment by Fafard et al. [29]. The smallest dots investigated there had a height less than $2 \mathrm{~nm}$ $(\approx 3.5 a)$ and the smallest distance $D$ between the WLs was $4 \mathrm{~nm}$. The most similar case in our study is $d=3 a$. Since the dots investigated in Ref. [29] were slightly larger than the QDs considered in the present work, the photoluminescence spectra revealed up to three or four peaks corresponding to three or four bound electron states. The red-shift observed for the lowest transition when going from $D=15 \mathrm{~nm}$ (well separated or single QD) to $D=4 \mathrm{~nm}$ was $\approx 23 \mathrm{meV}$. It fits well to the red-shift of $\approx 22 \mathrm{meV}$ calculated for the case $d=3 a$ (not shown in Fig. 6 but seen in Fig. 3). Also the red-shift of the WL threshold (see Fig. 3) agrees well with the experimental observations of Ref. [29].

\subsection{Strain effects}

Strain effects are well known to be important for self-assembled dots, but they are usually investigated within the continuous medium models $[8,9,13]$. Very recently strain effects in pyramidal InAs/GaAs quantum dots have been studied [15] using the TB and VFF approaches [30, 31]. Here we show how the strain effects influence the energy structure of single lens-shape InAs QD situated on a wetting layer and embedded in a GaAs cylinder. The size of dot and especially the size of the GaAs cylinder have to be larger than the ones considered in the previous sections. The InAs nanocrystal must be large enough to allow for strain and lattice relaxation inside the dot as it happens in real self-organized dots. The GaAs box should be taken as large as to minimize the effects of strain at the box boundaries. The diameter of the GaAs cylinder is now $22 a$; its height is $17 a$. The height of the lens is $3 a$ and its diameter is $12 a$. The investigated QD contains 1397 atoms (5203 with the WL) and the entire system contains 52526 atoms. 
TABLE

Several lowest electron and hole energy levels $(\mathrm{eV})$ for the unstrained and strained InAs QD. Zero of the energy scale is at the top of valence band of the unstrained system.

\begin{tabular}{c|c|c|c}
\hline \hline \multicolumn{2}{c|}{ Electron } & \multicolumn{2}{c}{ Hole } \\
\hline unstrained & strained & unstrained & strained \\
\hline 1.073 & 1.233 & -0.117 & -0.104 \\
1.072 & 1.212 & -0.102 & -0.092 \\
0.922 & 1.098 & -0.099 & -0.084
\end{tabular}
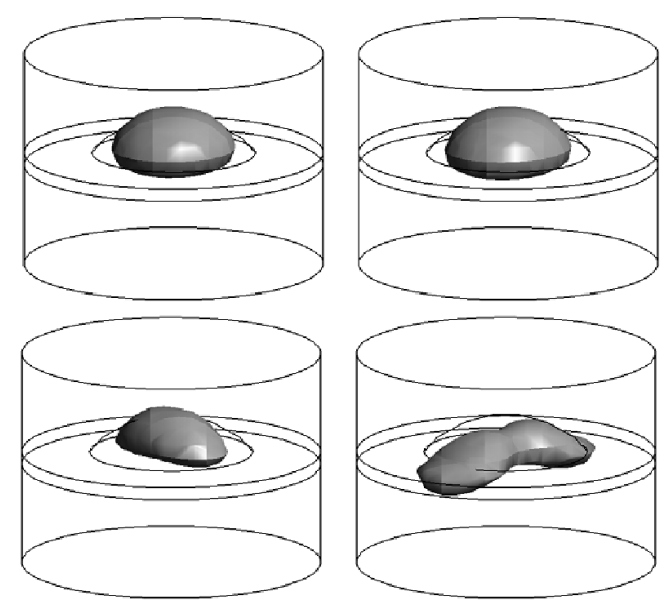

Fig. 7. Density isosurfaces (50\%) of the ground electron (top) and hole (bottom) states for the unstrained (left) and strained (right) InAs QD.

Several lowest electron and hole energy levels for unstrained and strained quantum dot are compared in Table. The unstrained structure contains 6 bound electron states localized inside the quantum dot. The number of bound hole states is 17. Closer inspection of charge densities for the case with the strain effects included reveals that there are only 3 bound and localized electron states inside the dot and only 10 well localized hole states. Charge densities of the ground electron and hole states for strained and unstrained dots are shown in Fig. 7. The electron states are less strain sensitive than the hole states. The main effect observed is that the hole ground state changes its space polarization and is weaker localized comparing with the unstrained case. As in Ref. [15] its density is strongly elongated along the (110) direction. This effect may significantly influence coupling between the dots in vertical stacks. Investigation of coupling and strain cross effects is above the scope of the present work and will be a subject of a separate paper. 


\section{Conclusions}

In conclusion, the tight-binding approach has been applied to describe the energy structure of two vertically stacked, InAs/GaAs, lens-shaped, self-assembled, quantum dots and to study atomic-scale effects on interdot coupling. Level degeneracies that are built into continuous medium models can be broken by atomic-scale effects. The degeneracy of the lowest single-dot hole state is lifted by atomic-scale effects. Level splitting due to atomic-scale symmetry breaking cannot be ignored because it is comparable to the level splitting due to tunneling between coupled dots.

Interdot coupling can be significant when the dots are separated by less than 10 ML. When the distance between the dots decreases, the coupling splits the energy levels and forms the bonding-like and antibonding-like states expected for an artificial molecule made from the two coupled dots. The splitting is more pronounced for electrons, but is more complex for holes. The complexity for holes is due to intradot and interdot mixing of the valence subbands that competes with the interdot tunneling and level alignment. We have found that the lowest double-dot hole state is a symmetric combination of single-dot hole states for closely spaced dots, but is an antisymmetric combination for widely spaced dots. These coupling effects change significantly the absorption spectra. For closely spaced dots, the lowest transition is optically active, but it becomes dark for widely spaced dots. Good agreement has been obtained between the calculated and measured red-shift of the lowest transition for decreasing distance between the coupled dots.

It has also been shown that strain effects lead to effectively shallower electron and hole wells and thus to weaker localization of states inside the quantum dot.

\section{Acknowledgments}

Financial support from KBN-3T11B04326 and PBZ-Min-008/P03/2003 is gratefully acknowledged.

\section{References}

[1] G.S. Solomon, J.A. Trezza, A.F. Marshall, J.S. Harris, Jr., Phys. Rev. Lett. 76, 952 (1996).

[2] P. Yu, W. Langbein, K. Leosson, J.M. Hvam, N.N. Ledentsov, D. Bimberg, V.M. Ustinov, A.Yu. Egorov, A.E. Zhukov, A.F. Tsatsul'nikov, Yu.G. Musikhin, Phys. Rev. B 60, 16680 (1999).

[3] B. Grandidier, Y.M. Niquet, B. Legrand, J.P. Nys, C. Priester, D. Stiévenard, J.M. Gérard, V. Thierry-Mieg, Phys. Rev. Lett. 85, 1068 (2000).

[4] M. Bayer, P. Hawrylak, K. Hinzer, S. Fafard, M. Korkusinski, Z.R. Wasilewski, O. Stern, A. Forchel, Science 291, 451 (2001).

[5] G.W. Bryant, Phys. Rev. B 40, 1620 (1989). 
[6] J.J. Palacios, P. Hawrylak, Phys. Rev. B 51, 1769 (1995).

[7] B. Szafran, S. Bednarek, J. Adamowski, Phys. Rev. B 64, 125301 (2001).

[8] A. Schliwa, O. Stier, R. Heitz, M. Grundmann, D. Bimberg, Phys. Status Solidi $B$ 224, 405 (2001).

[9] L.R.C. Fonseca, J.L. Jimenez, J.P. Leburton, Phys. Rev. B 58, 9955 (1998).

[10] M. Korkusiński, P. Hawrylak, Phys. Rev. B 63, 195311 (2001).

[11] S. Taddei, M. Colocci, A. Vinattieri, P.G. Gucciardi, F. Bogani, S. Franchi, P. Frigeri, L. Lazzarini, G. Salviati, Phys. Status Solidi B 224, 413 (2001).

[12] A. Vasanelli, M. De Giorgi, R. Ferreira, R. Cingolani, G. Bastard, Physica E 11, 41 (2001).

[13] M. Tadić, F.M. Peeters, B. Partoens, K.L. Janssens, Physica E 13, 237 (2002).

[14] W. Sheng, J.-P. Leburton, Phys. Rev. Lett. 88, 167401 (2002).

[15] R. Santoprete, Belita Koiller, R.B. Capaz, P. Kratzer, Q.K.K. Liu, M. Scheffler, Phys. Rev. B 68, 235311 (2003).

[16] P.E. Lippens, M. Lannoo, Phys. Rev. B 41, 6079 (1989).

[17] K. Leung, S. Pokrant, K.B. Whaley, Phys. Rev. B 57, 12291 (1998).

[18] G.W. Bryant, W. Jaskólski, Phys. Status Solidi B 224, 751 (2001).

[19] G.W. Bryant, W. Jaskólski, Physica E 11, 72 (2001).

[20] G.W. Bryant, W. Jaskólski, Phys. Rev. B 67, 205320 (2003).

[21] A. Mews, A.V. Kadavanich, U. Banin, A.P. Alivisatos, Phys. Rev. B 53, R13242 (1996).

[22] W. Jaskólski, G.W. Bryant, J. Planelles, M. Zieliński, Int. J. Quantum Chem. 90, 1075 (2002).

[23] H. Döllefeld, H. Weller, A. Eychmüller, Nano Lett. 1, 267 (2001).

[24] M.V. Artemyev, A.I. Bibik, L.I. Gurinovich, S.V. Gaponenko, U. Woggon, Phys. Rev. B 60, 1504 (1999).

[25] P. Vogl, H.P. Hjalmarson, J.D. Dow, J. Phys. Chem. Solids 44, 365 (1983).

[26] W.E. Arnoldi, Quart. J. Appl. Math. 9, 17 (1951); Y. Saad, Numerical Methods for Large Scale Eigenvalue Problems, Halsted Press, New York 1992; R.B. Morgan, Math. Comp. 65, 1213 (1996).

[27] O. Stier, M. Grundmann, D. Bimberg, Phys. Rev. B 59, 5688 (1999).

[28] S. Fraga, J. Muszyńska, Atoms in External Fields, Elsevier, New York 1981.

[29] S. Fafard, M. Spanner, J.P. McCaffrey, Z.R. Wasilewski, Appl. Phys. Lett. 76, 2268 (2000).

[30] C. Pryor, J. Kim, L.W. Wang, A.J. Williamson, A. Zunger, J. Appl. Phys. 83, 2548 (1998).

[31] T. Saito, Y. Arakawa, Physica E 15, 169 (2002). 